\title{
New limb-darkening coefficients for PHOENIX/1D model atmospheres
}

\section{Calculations for $5000 \mathrm{~K} \leq T_{\text {eff }} \leq 10000 \mathrm{~K}$ Kepler, CoRot, Spitzer, uvby, UBVRIJHK, Sloan, and 2MASS photometric systems * (Research Note)}

\author{
A. Claret ${ }^{1}$, P. H. Hauschildt ${ }^{2}$, and S. Witte ${ }^{2}$ \\ ${ }^{1}$ Instituto de Astrofísica de Andalucía, CSIC, Apartado 3004, 18080 Granada, Spain \\ e-mail: claret@iaa.es \\ 2 Hamburger Sternwarte, Gojenbergsweg 112, 21029 Hamburg, Germany
}

Received 18 December 2012 / Accepted 13 February 2013

ABSTRACT

\begin{abstract}
Aims. We present an extension of our investigations on limb-darkening coefficients computed with spherical symmetrical PHOENIX models. The models investigated in this paper cover the range $5000 \mathrm{~K} \leq T_{\text {eff }} \leq 10000 \mathrm{~K}$ and complete our previous studies of low effective temperatures computed with the same code.

Methods. The limb-darkening coefficients are computed for the transmission curves of the Kepler, CoRoT, and Spitzer space missions and the Strömgren, Johnson-Cousins, Sloan, and 2MASS passbands. These computations were performed by adopting the leastsquares method.

Results. We have used six laws to describe the specific intensity distribution: linear, quadratic, square root, logarithmic, exponential, and a general law with four terms. The computations are presented for the solar chemical composition and cover the range $3.0 \leq$ $\log g \leq 5.5$. The adopted microturbulent velocity and the mixing-length parameter are $2.0 \mathrm{~km} \mathrm{~s}^{-1}$ and 2.0 .
\end{abstract}

Key words. stars: atmospheres - binaries: eclipsing - planetary systems

\section{Introduction}

Some years ago, Claret \& Hauschildt (2003) presented calculations of limb-darkening coefficients (LDC) for spherical symmetrical PHOENIX models with $5000 \mathrm{~K} \leq T_{\text {eff }} \leq 10000 \mathrm{~K}$. At that occasion, the LDC were computed only for the classical photometric systems. Here we expand those calculations to the Kepler, CoRoT, and Spitzer space missions as well as to the Sloan and 2MASS passbands. For completeness, we also provide the calculations for the Strömgren and Johnson-Cousins photometric systems. This short paper completes our previous paper on LCD calculated with PHOENIX models (Claret et al. 2012), where we analysed models for late-type stars (1500 K $\leq$ $\left.T_{\text {eff }} \leq 4800 \mathrm{~K}\right)$.

\section{Limb-darkening coefficients for PHOENIX models}

For a description of the PHOENIX code that was used to compute the specific intensities, we refer to Claret \& Hauschildt (2003). The laws of limb-darkening used in the present paper are the linear law

$\frac{I(\mu)}{I(1)}=1-u(1-\mu)$

* Tables 2-25 are available in electronic form at the CDS via anonymous ftp to cdsarc.u-strasbg. fr (130.79.128.5) or via http://cdsarc.u-strasbg.fr/viz-bin/qcat?]/A+A/552/A16 the quadratic law

$\frac{I(\mu)}{I(1)}=1-a(1-\mu)-b(1-\mu)^{2}$,

the square root law

$\frac{I(\mu)}{I(1)}=1-c(1-\mu)-d(1-\sqrt{\mu})$,

the logarithmic law

$\frac{I(\mu)}{I(1)}=1-e(1-\mu)-f \mu \ln (\mu)$,

the exponential law

$\frac{I(\mu)}{I(1)}=1-g(1-\mu)-\frac{h}{\left(1-e^{\mu}\right)}$

and a more general law with four terms

$\frac{I(\mu)}{I(1)}=1-\sum_{k=1}^{4} a_{k}\left(1-\mu^{\frac{k}{2}}\right)$.

The symbols $u, a, b, c, d, e, f, g, h$, and $a_{k}$ denote the corresponding $\mathrm{LDC}$ and $\mu=\cos (\gamma), \gamma$ being the angle between the line of sight and the emerging intensity. The specific intensities were convolved with a response function containing the filter transmission curves for Kepler, CoRoT, Spitzer (IRAC), 

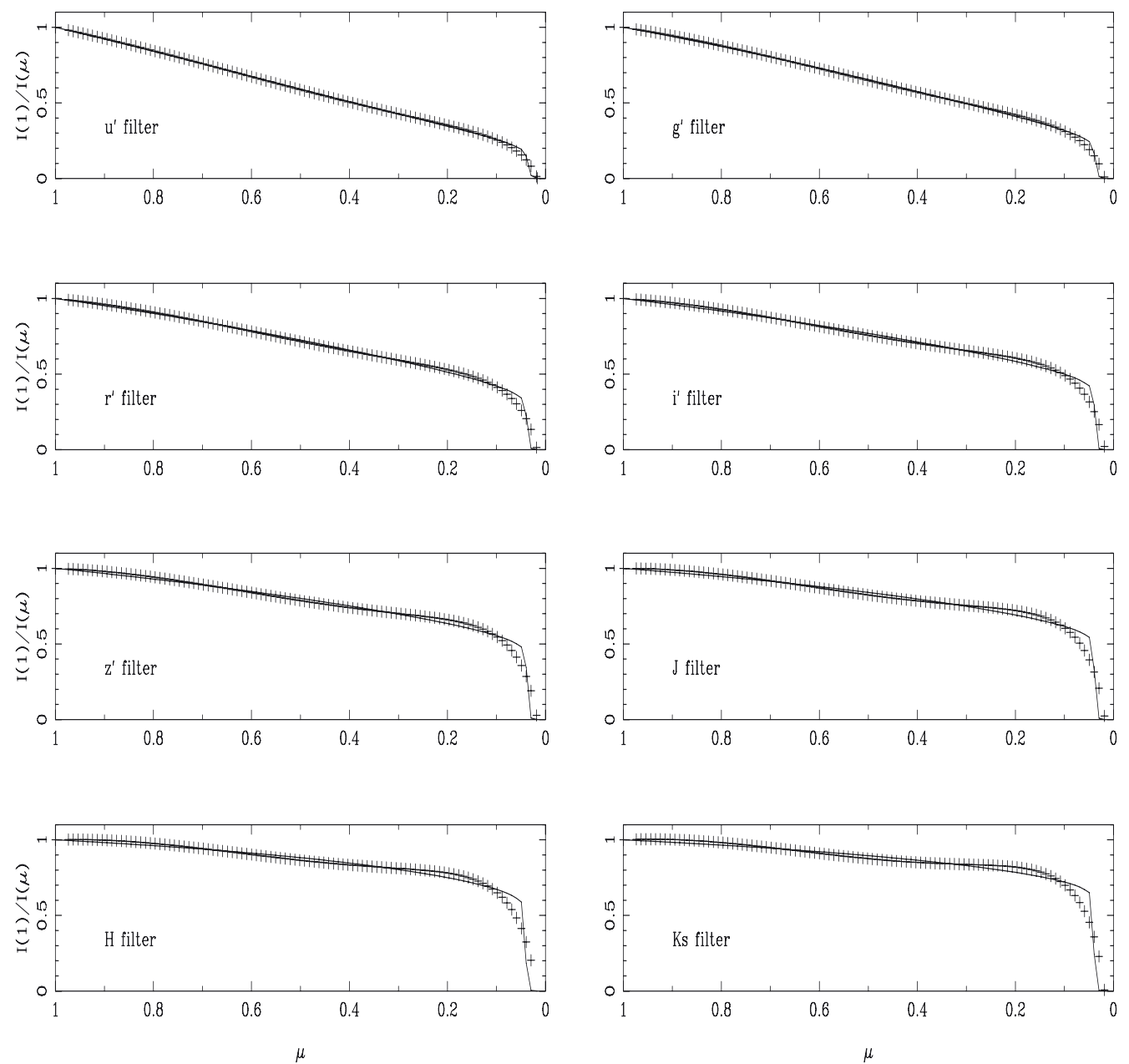

Fig. 1. Specific intensity distribution for a $[6000,4.5]$ model. Continuous line represents the actual intensities while crosses denote the fits by adopting Eq. (6). Sloan and 2MASS photometric systems.

uvby (Strömgren), UBVRIJHK (Johnson-Cousins), Sloan, and 2MASS, double reflection from an aluminium-coated mirror, and detector sensitivity. All calculations were performed by adopting the least-squares method (LSM). To provide users with a tool for evaluating the theoretical error bars in the LDC we also computed the coefficients with the flux conservation method (FCM) for the bi-parametric and linear approximations. For a more detailed description of the adopted numerical methods to derive the LDC, see Claret \& Hauschildt (2003) and Claret et al. (2012).

In Fig. 1 we show the resulting LDC for the Sloan and 2Mass photometric systems. The actual integrated intensities are compared with the fits provided by Eq. (6) for a model with $T_{\text {eff }}=6000 \mathrm{~K}$ and $\log g=4.5$. The inter-agreement is good, except for longer effective wavelengths, although they can be considered acceptable. This is due to the steeper profiles of the integrated specific intensities at drop-off zones for larger wavelengths. A similar pattern was also found for models with $1500 \mathrm{~K} \leq T_{\text {eff }} \leq 4800 \mathrm{~K}$ (Claret et al. 2012).

A quasi-spherical model is defined as the one computed with spherical symmetry but without considering the drop-off region. (for a more detailed definition of these models, see Claret et al. 2012). These models can be compared with previous calculations of linear LDC, such as those based on the plane-parallel ATLAS code (Kurucz, priv. comm.) This comparison is shown in Fig. 2. For the Kepler and Corot passbands we detect a systematic difference: the PHOENIX linear LDC are slightly larger than the corresponding to the ATLAS models. On the other hand, for Spitzer passbands, we notice an opposite effect: PHOENIX LDC are slightly smaller than those derived with the ATLAS code. These differences are approximately of the same order as the semi-empirical errors of the LDC. It would be useful if observers could compare the LDC provided by PHOENIX and ATLAS models with the objective of providing some clues to the stellar atmosphere modellers so that they in turn can improve the theoretical models. Despite these systematic effects, the inter-agreement is good. The discontinuities in the linear LDC around $\log T_{\text {eff }}=3.9$ for both models are also notorious. These small jumps are characteristic of the atmosphere models and are connected to the onset of convection (PHOENIX and ATLAS adopted 2.0 and 1.25 for the mixing-length parameter, respectively). These discontinuities in the linear LDC are dependent on the passband, which are smaller for the larger effective wavelengths.

On the other hand, it is known that it is very difficult to observationally detect the low intensities near the extreme limb predicted by spherical models. As explained in Claret et al. (2012), only bi-parametric semi-empirical LDC can be, at best, inferred by using high-quality light curves of extra-solar planet transits or double-lined eclipsing binaries, for example. However, the intensity profiles of the spherical models are complicated and the bi-parametric laws are not able to reproduce these profiles well, except for the exponencial law (Eq. (5)). Therefore, the concept of quasi-spherical models is very useful in these cases since their 
A. Claret et al.: limb-darkening coefficients. II. $(R N)$
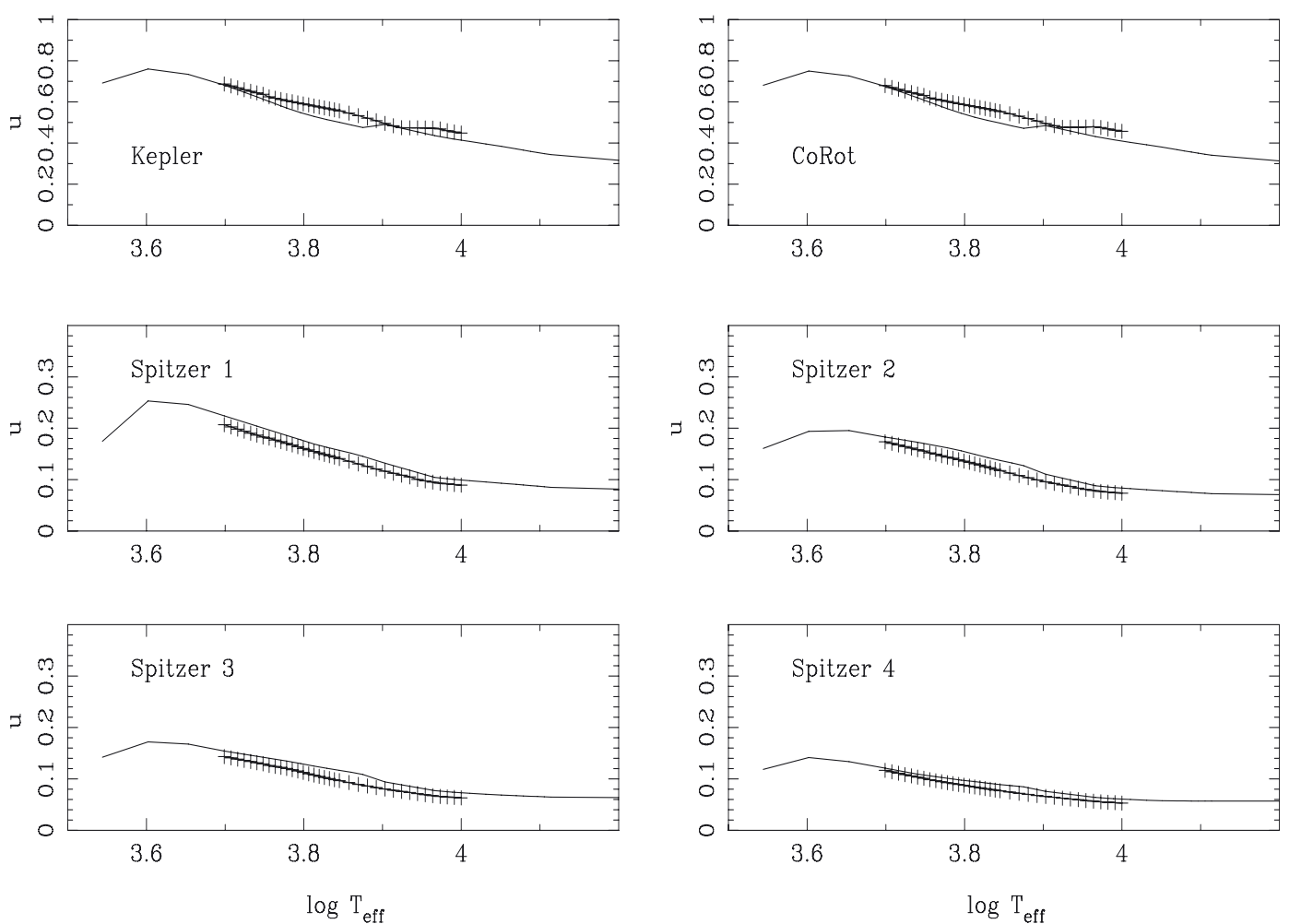

Fig. 2. Linear LDC for quasi-spherical PHOENIX calculations (crosses) and ATLAS plane-parallel models (continuous lines). Log $g=4.0$, solar composition, Kepler, CoRot, and Spitzer passbands.

Table 1. Limb-darkening coefficients for the Kepler, CoRoT, Spitzer, UBVRIJHK, uvby, Sloan, and 2MASS photometric systems.

\begin{tabular}{|c|c|c|c|c|c|c|c|}
\hline Name & Source & Range $T_{\text {eff }}$ & Range $\log g$ & $\log [\mathrm{M} / \mathrm{H}]$ & Vel Turb. & Filter & Fit/equation \\
\hline Table 2 & PHOENIX & $5000 \mathrm{~K}-10000 \mathrm{~K}$ & $3.0-5.5$ & 0.0 & $2 \mathrm{~km} \mathrm{~s}^{-1}$ & Kepler, CoRot, Spitzer & LSM/FCM/Eq. (1) quasi-spherical \\
\hline Table 3 & PHOENIX & $5000 \mathrm{~K}-10000 \mathrm{~K}$ & $3.0-5.5$ & 0.0 & $2 \mathrm{~km} \mathrm{~s}^{-1}$ & Kepler, CoRot, Spitzer & LSM/FCM/Eq. (2) quasi-spherical \\
\hline Table 4 & PHOENIX & $5000 \mathrm{~K}-10000 \mathrm{~K}$ & $3.0-5.5$ & 0.0 & $2 \mathrm{~km} \mathrm{~s}^{-1}$ & Kepler, CoRot, Spitzer & LSM/FCM/Eq. (3) quasi-spherical \\
\hline Table 5 & PHOENIX & $5000 \mathrm{~K}-10000 \mathrm{~K}$ & $3.0-5.5$ & 0.0 & $2 \mathrm{~km} \mathrm{~s}^{-1}$ & Kepler, CoRot, Spitzer & LSM/FCM/Eq. (4) quasi-spherical \\
\hline Table 6 & PHOENIX & $5000 \mathrm{~K}-10000 \mathrm{~K}$ & $3.0-5.5$ & 0.0 & $2 \mathrm{~km} \mathrm{~s}^{-1}$ & Kepler, CoRot, Spitzer & LSM/Eq. (5) quasi-spherical \\
\hline Table 7 & PHOENIX & $5000 \mathrm{~K}-10000 \mathrm{~K}$ & $3.0-5.5$ & 0.0 & $2 \mathrm{~km} \mathrm{~s}^{-1}$ & Kepler, CoRot, Spitzer & LSM/Eq. (6) quasi-spherical \\
\hline Table 8 & PHOENIX & $5000 \mathrm{~K}-10000 \mathrm{~K}$ & $3.0-5.5$ & 0.0 & $2 \mathrm{~km} \mathrm{~s}^{-1}$ & Kepler, CoRot, Spitzer & LSM/Eq. (5) spherical \\
\hline Table 9 & PHOENIX & $5000 \mathrm{~K}-10000 \mathrm{~K}$ & $3.0-5.5$ & 0.0 & $2 \mathrm{~km} \mathrm{~s}^{-1}$ & Kepler, CoRot, Spitzer & LSM/Eq. (6) spherical \\
\hline Table 10 & PHOENIX & $5000 \mathrm{~K}-10000 \mathrm{~K}$ & $3.0-5.5$ & 0.0 & $2 \mathrm{~km} \mathrm{~s}^{-1}$ & uvbyUBVRIJHK & LSM/FCM/Eq. (1) quasi-spherical \\
\hline Table 11 & PHOENIX & $5000 \mathrm{~K}-10000 \mathrm{~K}$ & $3.0-5.5$ & 0.0 & $2 \mathrm{~km} \mathrm{~s}^{-1}$ & uvbyUBVRIJHK & LSM/FCM/Eq. (2) quasi-spherical \\
\hline Table 12 & PHOENIX & $5000 \mathrm{~K}-10000 \mathrm{~K}$ & $3.0-5.5$ & 0.0 & $2 \mathrm{~km} \mathrm{~s}^{-1}$ & uvbyUBVRIJHK & LSM/FCM/Eq. (3) quasi-spherical \\
\hline Table 13 & PHOENIX & $5000 \mathrm{~K}-10000 \mathrm{~K}$ & $3.0-5.5$ & 0.0 & $2 \mathrm{~km} \mathrm{~s}^{-1}$ & uvbyUBVRIJHK & LSM/FCM/Eq. (4) quasi-spherical \\
\hline Table 14 & PHOENIX & $5000 \mathrm{~K}-10000 \mathrm{~K}$ & $3.0-5.5$ & 0.0 & $2 \mathrm{~km} \mathrm{~s}^{-1}$ & uvbyUBVRIJHK & LSM/Eq. (5) quasi-spherical \\
\hline Table 15 & PHOENIX & $5000 \mathrm{~K}-10000 \mathrm{~K}$ & $3.0-5.5$ & 0.0 & $2 \mathrm{~km} \mathrm{~s}^{-1}$ & uvbyUBVRIJHK & LSM/Eq. (6) quasi-spherical \\
\hline Table 16 & PHOENIX & $5000 \mathrm{~K}-10000 \mathrm{~K}$ & $3.0-5.5$ & 0.0 & $2 \mathrm{~km} \mathrm{~s}^{-1}$ & uvbyUBVRIJHK & LSM/Eq. (5) spherical \\
\hline Table 17 & PHOENIX & $5000 \mathrm{~K}-10000 \mathrm{~K}$ & $3.0-5.5$ & 0.0 & $2 \mathrm{~km} \mathrm{~s}^{-1}$ & uvbyUBVRIJHK & LSM/Eq. (6) spherical \\
\hline Table 18 & PHOENIX & $5000 \mathrm{~K}-10000 \mathrm{~K}$ & $3.0-5.5$ & 0.0 & $2 \mathrm{~km} \mathrm{~s}^{-1}$ & Sloan, 2MASS & LSM/FCM/Eq. (1) quasi-spherical \\
\hline Table 19 & PHOENIX & $5000 \mathrm{~K}-10000 \mathrm{~K}$ & $3.0-5.5$ & 0.0 & $2 \mathrm{~km} \mathrm{~s}^{-1}$ & Sloan, 2MASS & LSM/FCM/Eq. (2) quasi-spherical \\
\hline Table 20 & PHOENIX & $5000 \mathrm{~K}-10000 \mathrm{~K}$ & $3.0-5.5$ & 0.0 & $2 \mathrm{~km} \mathrm{~s}^{-1}$ & Sloan, 2MASS & LSM/FCM/Eq. (3) quasi-spherical \\
\hline Table 21 & PHOENIX & $5000 \mathrm{~K}-10000 \mathrm{~K}$ & $3.0-5.5$ & 0.0 & $2 \mathrm{~km} \mathrm{~s}^{-1}$ & Sloan, 2MASS & LSM/FCM/Eq. (4) quasi-spherical \\
\hline Table 22 & PHOENIX & $5000 \mathrm{~K}-10000 \mathrm{~K}$ & $3.0-5.5$ & 0.0 & $2 \mathrm{~km} \mathrm{~s}^{-1}$ & Sloan, 2MASS & LSM/Eq. (5) quasi-spherical \\
\hline Table 23 & PHOENIX & $5000 \mathrm{~K}-10000 \mathrm{~K}$ & $3.0-5.5$ & 0.0 & $2 \mathrm{~km} \mathrm{~s}^{-1}$ & Sloan, 2MASS & LSM/Eq. (6) quasi-spherical \\
\hline Table 24 & PHOENIX & $5000 \mathrm{~K}-10000 \mathrm{~K}$ & $3.0-5.5$ & 0.0 & $2 \mathrm{~km} \mathrm{~s}^{-1}$ & Sloan, 2MASS & LSM/Eq. (5) spherical \\
\hline Table 25 & PHOENIX & $5000 \mathrm{~K}-10000 \mathrm{~K}$ & $3.0-5.5$ & 0.0 & $2 \mathrm{~km} \mathrm{~s}^{-1}$ & Sloan, 2MASS & LSM/Eq. (6) spherical \\
\hline
\end{tabular}

theoretical bi-parametric (or linear) LDC can be directly compared with the semi-empirical ones.

In Table 1 we summarise the results for the LDC calculations (Tables 2-25). These tables can be downloaded directly from CDS. Additional calculations for other photometric systems not covered in this paper can be provided on request.

Acknowledgements. The Spanish MEC (AYA2006-06375, AYA2009-14000C03-01) is gratefully acknowledged for its support during the development of this work. This research has made use of the SIMBAD database, operated at the CDS, Strasbourg, France, and of NASA's Astrophysics Data System Abstract Service.

\section{References}

Claret, A., \& Hauschildt, P. H. 2003, A\&A, 412, 241

Claret, A., Hauschildt, P. H., \& Witte, S. 2012, A\&A, 546, A14 